\title{
A Collision Tumor With Features of Breast Cancer and Plasma Cell Myeloma as Primary Tumors
}

\author{
Leila Moosavia, c, Sassan Farjami ${ }^{a}$, Lisa Shane ${ }^{a}$, Alireza Hosseini ${ }^{b}$, \\ Chester Choi ${ }^{\mathrm{a}}$
}

\begin{abstract}
Collision tumors, consisting of two different histological types of cancer, are rare but may have important implications, both for the patient and possibly in terms of cancer risks and predisposition. This patient has had prior invasive ductal breast cancer treated with surgery and chemotherapy 5 years earlier, but she developed a pathological hip fracture and anemia. The workup uncovered a plasma cell dyscrasia and the bone biopsy from the hip showed the collision tumor with an interface between breast cancer and plasma cell cancer. Such combination tumors may be diagnostic and therapeutic challenges, but they also raise significant questions regarding pathogenesis. Local factors such as neuro-immune crosstalk or cytokine perturbations may be at play. Alternatively, or in addition, oncogenic growth factors or the effects of prior chemotherapy may be significant.
\end{abstract}

Keywords: Collision tumor; Histopathology; Monoclonal gammopathy; Plasma cell dyscrasia; Lytic/blastic bone lesions; Genetic predisposition

\section{Introduction}

Collision tumors are defined by the presence of cancers of different histopathological origin at the same anatomical organ or site. The diagnosis of this very rare type of tumor depends upon histopathology and also needs careful review of morphology, immunohistochemistry staining, and specific cytogenetics. While collision tumors of other histopathologies have been described in multiple locations, including the gastric cardia, uterine cervix, renal pelvis, bladder, liver, lung, oral cavity, thyroid, ovary and bile ducts, this is, to our knowledge, the first reported case of a collision tumor consisting of both breast cancer and multiple myeloma found in

Manuscript submitted June 10, 2018, accepted July 24, 2018

aSt. Mary Medical Center, Long Beach, CA 90813, USA

bIran University of Medical Sciences, Tehran, Iran

${ }^{\mathrm{c} C o r r e s p o n d i n g ~ A u t h o r: ~ L e i l a ~ M o o s a v i, ~ D e p a r t m e n t ~ o f ~ I n t e r n a l ~ M e d i c i n e, ~}$ St. Mary Medical Center, 1050 Linden Ave., Long Beach, CA 90813, USA. Email: lilimoosavi@gmail.com

doi: https://doi.org/10.14740/jmc3117w bone [1-11]. The pathogenesis of such combination tumors is unclear; they may be simply unfortunate and rare dual afflictions, but may alternatively be due to genetic, cellular, cytokinetic, or other perturbations, local or systemic. The diagnosis and treatment of such dual tumors may be more complex and may require careful planning and timing of multiple anti-cancer modalities to maximize their effects and minimize complications.

\section{Case Report}

This 79-year-old woman had a history of right breast invasive ductal carcinoma stage IIB, estrogen receptor (ER) and progesterone receptor (PR) positive, human epidermal growth factor receptor 2 (HER2/neu) negative by FISH/IHC and, was originally treated with modified radical right mastectomy followed by adjuvant chemotherapy and adjuvant aromatase inhibitor. She failed regular follow-up with her oncologist but was living independently and had full functional status prior to this hospitalization. Five years after her original diagnosis, she presented to the emergency department with excruciating right hip pain after sustaining a ground level fall. Computerized tomography (CT) of the pelvis revealed a compound fracture of the right femoral neck and a displaced fracture of the left sacral ala suggestive for pathological fracture (Fig. 1). She had a microcytic, hypochromic anemia and elevated total protein and serum globulins on admission (Table 1). She underwent right hip open reduction and internal fixation and a bone biopsy obtained from the area during surgery showed metastatic breast carcinoma which was positive for GATA3 (Fig. 2), strongly positive for ER and PR (Fig. 3) but HER2/ neu negative. In addition to metastatic breast carcinoma, the adjacent bone marrow showed an abnormal population of plasma cells positive for CD138 (Fig. 4) and representing approximately $80 \%$ of the total cellularity of the marrow. Serum and urine electrophoresis, serum and urine immunofixation, and free light chain assays were done as part of the plasma cell dyscrasia workup. The results revealed an abnormal $\operatorname{IgG}$ kappa monoclonal protein elevated to $5,300.0 \mathrm{mg} / \mathrm{dL}$ with elevated kappa-lambda light chain ratio (K/L) 2,406.90 (Table 2). Additional immunohistochemical stains were performed and showed negative CD56 and clonality positive for kappa but no staining for lambda light chains. These findings were all consistent with involvement of bone by malignant clonal plasma cells as well. Given this dual diagnosis of metastatic 


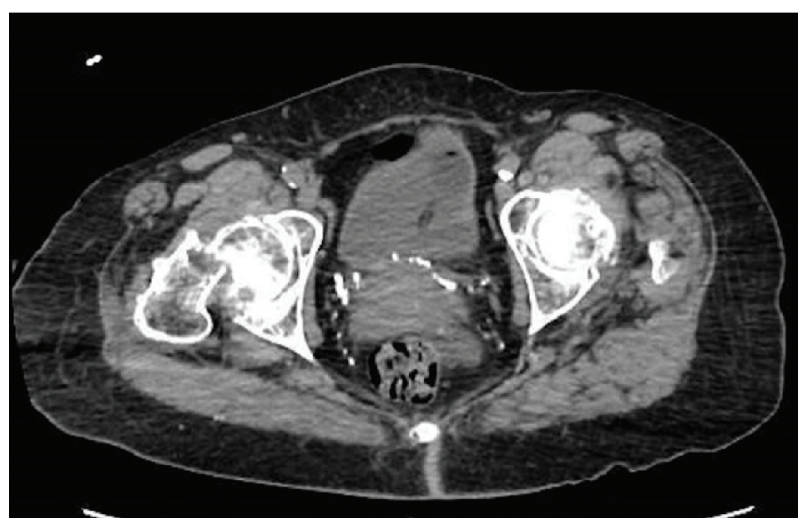

Figure 1. Imaging CT hip: right femoral neck fracture with nondisplaced fracture of the left sacral ala.

breast cancer and multiple myeloma, additional workup was initiated to determine the stages of these diseases. She underwent a Tc $99 \mathrm{~m}$ nuclear medicine bone scan as a part of staging for breast cancer which revealed increased radiotracer activity in the left frontal skull, the posterior aspect of the left 10th rib at the costovertebral junction, the thoracic (T3) vertebral body, and also left sacroiliac joint. CT of the chest and abdomen revealed enlargement of the pericarinal, subcarinal, and right hilar nodes as well as multiple pleural-based masses in the right lung consistent with metastatic disease. No evidence of metastatic disease was detected in the liver, spleen or adrenal glands. Bone marrow biopsy was deferred given the above results of the surgical specimen.

\section{Treatment}

The findings were compatible with a collision tumor of breast cancer (stage IV) and multiple myeloma (stage II by the international staging system) of the right hip (Fig. 2); both were felt to be radiosensitive malignancies. Palliative radiotherapy followed by chemotherapy with an aromatase inhibitor initiated for metastatic breast carcinoma and first cycle of induction chemotherapy with the combination of a proteasome inhibitor, cyclophosphamide and dexamethasone (CyborD) was started for the newly diagnosed multiple myeloma. It was felt that the alkylating agents such as cyclophosphamide would also target the metastatic breast cancer. The patient tolerated the first cycle of chemotherapy well without significant complications and was discharged home on continued anastrozole with plans for a second cycle as an out-patient.

\section{Discussion}

Combination tumors of different histology are divided into two pathological groups: collision and composite tumors. Collision tumors have two or more juxtapositioned masses and each mass has a distinct boundary, whereas composite tumors have intermingled cell types. Collision of two or even more
Table 1. Laboratory Results on Admission

\begin{tabular}{|c|c|}
\hline \multicolumn{2}{|l|}{$\mathrm{CBC}$} \\
\hline White blood cell count & 6.3 \\
\hline Neutrophils & 66 \\
\hline Lymphocytes & 21 \\
\hline Monocytes & $12(\mathrm{H})$ \\
\hline Red blood cell count & $3.09(\mathrm{~L})$ \\
\hline Hemoglobin & $8.1(\mathrm{~L})$ \\
\hline Hematocrit & $25.6(\mathrm{~L})$ \\
\hline $\mathrm{MCV}$ & 83.0 \\
\hline RDW & $19.3(\mathrm{H})$ \\
\hline Platelets & 187 \\
\hline \multicolumn{2}{|l|}{ LFT and coagulation factors } \\
\hline $\mathrm{AKP}$ & 68 \\
\hline ALT & 10 \\
\hline AST & $53(\mathrm{H})$ \\
\hline Bilirubin & 0.5 \\
\hline ALB & $2.5(\mathrm{~L})$ \\
\hline Protein & $9.8(\mathrm{H})$ \\
\hline Globulin & $7.3(\mathrm{H})$ \\
\hline PT & $15.5(\mathrm{H})$ \\
\hline APTT & 28.8 \\
\hline INR & $1.23(\mathrm{H})$ \\
\hline \multicolumn{2}{|l|}{ BMP } \\
\hline Glucose & $100(\mathrm{H})$ \\
\hline BUN & 20 \\
\hline Creatinine & 0.65 \\
\hline Sodium & $130(\mathrm{~L})$ \\
\hline Chloride & 100 \\
\hline Potassium & 4.4 \\
\hline Calcium & 9.3 \\
\hline $\mathrm{CO}_{2}$ & 22 \\
\hline \multicolumn{2}{|l|}{ Tumor marker } \\
\hline CEA & $<1$ \\
\hline
\end{tabular}

CBC: complete blood count; MCV: mean corpuscular volume; RDW: red blood cell distribution; AKP: alkaline phosphatase; ALT: alanine amino transferase; AST: aspartate aminotransferase; ALB: albumin; PT: prothrombin time; APTT: activated partial thromboplastin time; INR: international normalized ratio; LFT: liver function test; BMP: basic metabolic panel; CEA: carcinoembryonic antigen; L: low; H: high.

malignancies can occur between cancers originating in the same organ or with metastases from other sites [1]. Bone is a well-known sanctuary site for metastatic breast cancer and is a primary site for multiple myeloma involvement. Both can certainly result in skeletal-related events such as the pathological fractures experienced by our patient.

The cause of collision tumors such as that in our patient 

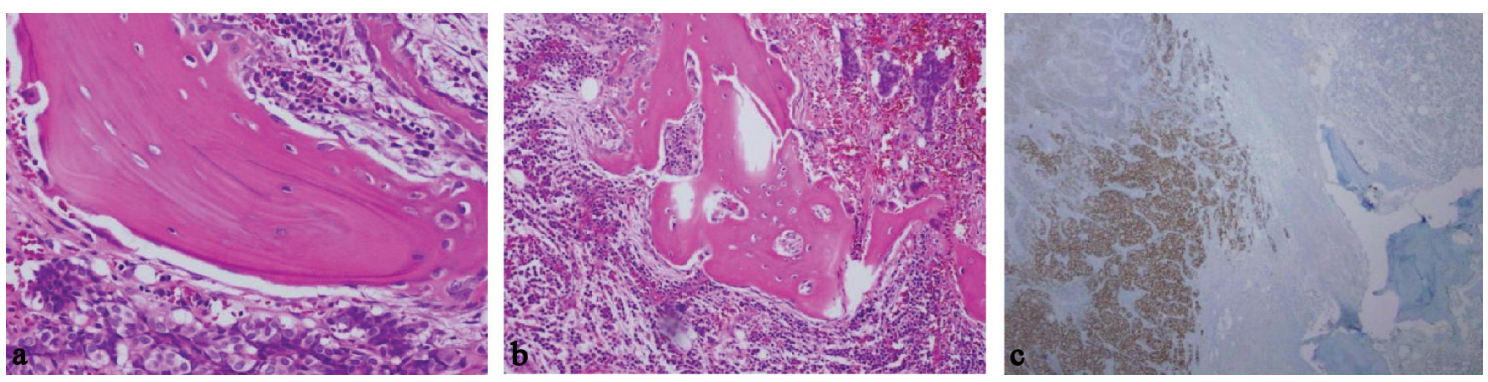

Figure 2. Breast cancer cells in bone marrow. (a) Presence of cells with breast origin, high power field. (b) Presence of plasma cells and breast cells, medium power field. (c) GATA 3 staining as marker of breast cancer.

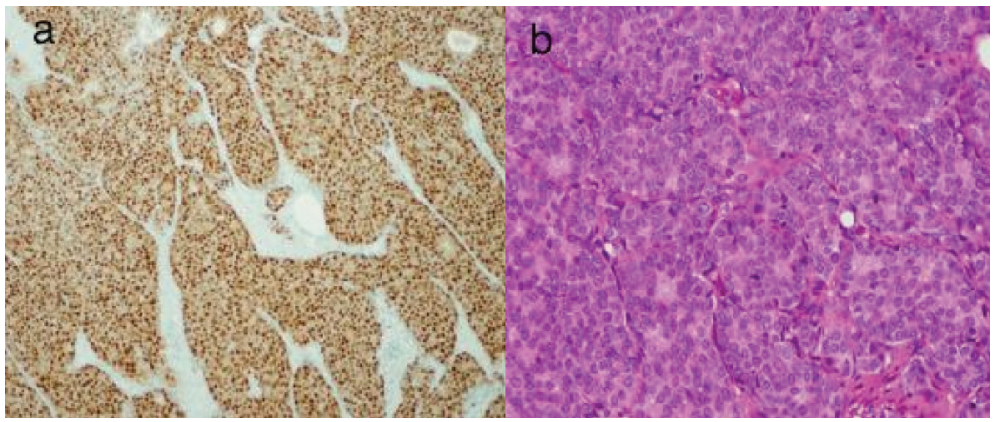

Figure 3. Sections of femoral head with metastatic breast cancer. (a) Strongly positive for estrogen receptor. (b) Strongly positive for progesterone receptor.

is unclear. Of course, coincidence is a possibility with the unfortunate meeting of two coexisting neoplasms developing independently and finally colliding [12]. This theory does not provide any particular explanation of the colliding pattern and makes no differentiation between composite and collision neoplasms [12]. Other possibilities seem more intriguing.

Our patient was previously treated with systemic chemotherapy and local breast radiation therapy 5 years prior to presenting with her multiple myeloma; thus, an explanation may be that chemotherapeutic medications used to treat a previous cancer may trigger the development of a second primary cancer. A second potential explanation is the "seeds and soil" theory in which cancer cells are the "seeds" and the specific organ microenvironment is the "soil". Interaction between the "seeds" and the "soil" may determine the formation of a secondary tumor [13]. The presence of metastatic breast cancer and its interaction on cytokines or its elaboration of growth factors may create a fertile microenvironment for second malignancies. Crosstalk between cancer cells is another possible explanation. Cancer cells can influence their microenvironment and bi-directionally communicate with other systems including the immune and nervous systems. While the immune system is well-recognized for its cancer regulatory function, the nervous system may also play a fundamental role in influencing immune responses to a range of disease states including cancer development and progression [14]. Finally, another possible explanation is that genetic predisposition or alteration may increase the likelihood of the development of two different cancers.

\section{Conclusions}

Our case is that of an unusual collision tumor with breast can-

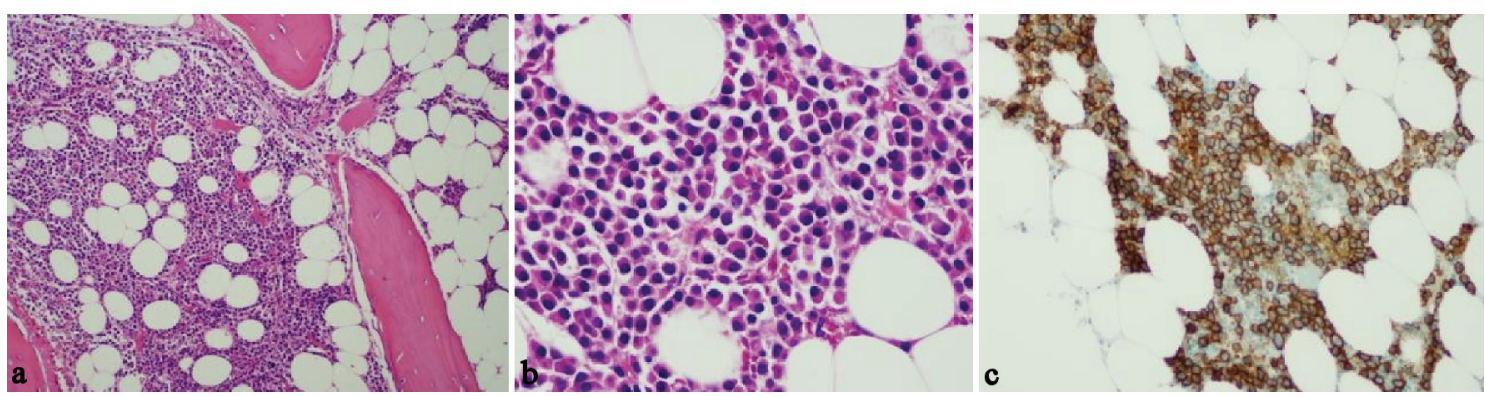

Figure 4. Morphology evaluation of bone marrow. (a) Hyper-cellular bone marrow. (b) Plasma cells intermixed with few small lymphocytes. (c) CD138 positive. 
Table 2. More Diagnostic Data Suggestive for Plasma Cell Dyscrasia

\begin{tabular}{|ll|}
\hline SPEP & $10.3(\mathrm{H})$ \\
\hline Total protein & 3.2 \\
\hline Albumin & $0.5(\mathrm{H})$ \\
\hline Alpha 1 & 0.9 \\
Alpha 2 & 0.9 \\
Beta & 4.7 \\
\hline Gamma & 3.5 \\
B2 microglobulin & \\
\hline Free light chain & $698.0(\mathrm{H})$ \\
\hline Kappa & $0.29(\mathrm{~L})$ \\
\hline Lambda & $2,406.90$ \\
\hline K/L ratio & \\
\hline Immunofixation & $<19$ \\
\hline IgA & $5,300(\mathrm{H})$ \\
\hline IgG & $<8$ \\
\hline IgM & \\
\hline
\end{tabular}

SPEP: serum protein electrophoresis; IgA: immunoglobulin A; IgG: immunoglobulin G; IgM: immunoglobulin M; Alpha 1: alpha-1 globulin; Alpha 2: alpha-2 globulin; Beta: beta globulin; Gamma: gamma globulin.

cer and plasma cell myeloma as the primary tumors. To the best of our knowledge, this is the first reported case of a collision tumor of this type involving bone. Collision tumors of other types have been described in multiple other locations; these bring forth questions regarding the pathogenesis and predisposing factors for such combination tumors. They also raise significant treatment concerns that require careful therapeutic choices regarding combination chemotherapy timing, and the use of other treatment modalities.

\section{Conflict of Interest}

The authors declare no conflict of interest in preparing this article.

\section{Disclosures}

The above case has been presented as poster presentation at the American College of Physicians Southern California Regional Scientific Meeting in September 2017.

\section{References}

1. Murthaiah P, Truskinovsky AM, Shah S, Dudek AZ. Collision tumor versus multiphenotypic differentiation: a case of carcinoma with features of colonic and lung primary tumors. Anticancer Res. 2009;29(5):1495-1497.

2. Wanke M. Collision-tumour of the cardia. Virchows Arch A Pathol Pathol Anat. 1972;357(1):81-86.

3. Spagnolo DV, Heenan PJ. Collision carcinoma at the esophagogastric junction: report of two cases. Cancer. 1980;46(12):2702-2708.

4. Lovell MO, Valente PT. Unique collision of hodgkin lymphoma and adenosquamous carcinoma in the uterine cervix: synchronous malignant neoplasms of the cervix. J Low Genit Tract Dis. 2003;7(4):307-310.

5. Phan ST, Meng M, Weidner N. Collision tumor: a peripheral neuroepithelioma and a transitional-cell carcinoma occurring simultaneously in the renal pelvis. Ann Diagn Pathol. 1997;1(2):91-98.

6. Garcia MT, Bejarano PA, Yssa M, Buitrago E, Livingstone A. Tumor of the liver (hepatocellular and high grade neuroendocrine carcinoma): a case report and review of the literature. Virchows Arch. 2006;449(3):376-381.

7. Lee AH, Soomro IN. Collision tumour of the pleura composed of small cell carcinoma and malignant mesothelioma. Histopathology. 2004;45(3):305-306.

8. Sirsat MV, Shrikhande SS. Collision tumour in the oral cavity (a report of 2 cases). Indian J Pathol Bacteriol. 1966;9(4):340-343.

9. Brandwein-Gensler M, Urken M, Wang B. Collision tumor of the thyroid: a case report of metastatic liposarcoma plus papillary thyroid carcinoma. Head Neck. 2004;26(7):637-641.

10. Nirenberg A, Ostor AG, Quinn MA. Collision tumor: serous adenocarcinoma and steroid cell tumor of the ovary. Pathology. 1992;24(2):60-62.

11. Hirono S, Tani M, Terasawa H, Kawai M, Ina S, Uchiyama K, Nakamura Y, et al. A collision tumor composed of cancers of the bile duct and ampulla of Vater - immunohistochemical analysis of a rare entity of double cancer. Hepatogastroenterology. 2008;55(84):861-864.

12. Michalinos A, Constantinidou A, Kontos M. Gastric collision tumors: an insight into their origin and clinical significance. Gastroenterol Res Pract. 2015;2015:314158.

13. Chao-Nan Qian, Bin Tean. The: "seed and soil" Theory of Metastasis, encyclopedia of cancer, 978-3-642-16483-5, 2017.

14. Kuol N, Stojanovska L, Apostolopoulos V, Nurgali K. Crosstalk between cancer and the neuro-immune system. J Neuroimmunol. 2018;315:15-23. 